\title{
Pawedness Trait Test (PaTRaT) - A New Paradigm to Evaluate Paw Preference and Dexterity in Rats
}

\author{
Ana M. Cunha ${ }^{1,2 \dagger}$, Madalena Esteves ${ }^{1,2 \dagger}$, Sofia P. das Neves ${ }^{1,2}$, Sónia Borges ${ }^{1,2}$, \\ Marco R. Guimarães ${ }^{1,2}$, Nuno Sousa ${ }^{1,2}$, Armando Almeida ${ }^{1,2}$ and Hugo Leite-Almeida ${ }^{1,2 *}$ \\ 1 Life and Health Sciences Research Institute (ICVS), School of Medicine, University of Minho, Braga, Portugal, \\ ${ }^{2}$ ICVS/3B's - PT Government Associate Laboratory, Braga, Portugal
}

OPEN ACCESS

Edited by:

Allan V. Kalueff,

St. Petersburg State University,

Russia

Reviewed by:

Giovanni Laviola,

Istituto Superiore di Sanità, Italy

Barbara Dotto Fontana,

Universidade Federal de Santa Maria,

Brazil

*Correspondence:

Hugo Leite-Almeida

hugoalmeida@med.uminho.pt

${ }^{\dagger}$ These authors have contributed equally to this work.

Received: 02 August 2017 Accepted: 30 September 2017

Published: 16 October 2017

Citation:

Cunha AM, Esteves M. das Neves SP, Borges S,

Guimarães MR, Sousa N, Almeida A and Leite-Almeida $H$ (2017) Pawedness Trait Test (PaTRaT)-A New Paradigm to

Evaluate Paw Preference and

Dexterity in Rats.

Front. Behav. Neurosci. 11:192. doi: 10.3389/fnbeh.2017.00192
In rodents, dexterity is commonly analyzed in preference paradigms in which animals are given the chance to use either the left or the right front paws to manipulate food. However, paw preference and dexterity at population and individual levels are controversial as results are incongruent across paradigms. We have therefore developed a semi-quantitative method-the pawdeness trait test (PaTRaT)-to evaluate paw preference degree in rats. The PaTRaT consists in a classification system, ranging from +4 to -4 where increasingly positive and negative values reflect the bias for left or right paw use, respectively. Sprague-Dawley male rats were confined into a metal rectangular mesh cylinder, from which they can see, smell and reach sugared rewards with their paws. Due to its size, the reward could only cross the mesh if aligned with its diagonal, imposing additional coordination. Animals were allowed to retrieve 10 rewards per session in a total of four sessions while their behavior was recorded. PaTRaT was repeated 4 and 8 weeks after the first evaluation. To exclude potential bias, rats were also tested for paw fine movement and general locomotion in other behavioral paradigms as well as impulsivity (variable delay-to-signal, VDS), memory and cognitive flexibility (water maze). At the population level 54\% of the animals presented a rightward bias. Individually, all animals presented marked side-preferences, $>2$ and $<-2$ for left- and right-sided bias, respectively, and this preference was stable across the three evaluations. Inter-rater consistency was very high between two experienced raters and substantial when two additional inexperienced raters were included. Left- and right-biased animals presented no differences in the ability to perform fine movements with any of the forelimbs (staircase) and general locomotor performance. Additionally, these groups performed similarly in executive function and memory tasks. In conclusion, PaTRaT is able to reliably classify rats' pawedness direction and degree.

Keywords: motor preference, laterality, handedness, cognition, impulsivity, memory, behavior

\section{INTRODUCTION}

Pawedness reflects the preferential use and/or an increased capacity to perform tasks more efficiently with a specific paw. It corresponds, in general terms, to animals' handedness. Pawedness/handedness is thought to be associated with brain asymmetries, present both at morphological, cellular and molecular levels (see for reviews 
Galaburda et al., 1978; Toga and Thompson, 2003; Sun and Walsh, 2006; Rogers, 2009, 2014; Hugdahl, 2011). Regarding morphology most studies have so far excluded any association (Good et al., 2001; Narr et al., 2007; Guadalupe et al., 2014, 2016; Ocklenburg et al., 2016); however at cellular and molecular levels, contralateral parietal spine density has been linked to skilled reaching (Ambeskovic et al., 2017) and dopaminergic system lateralization has been shown to be associated with hand/paw preference in humans (de la Fuente-Fernández et al., 2000) as well as in rodents (UguruOkorie and Arbuthnott, 1981; Schwarting et al., 1987; Barnéoud et al., 1990; Cabib et al., 1995; Nielsen et al., 1997; Budilin et al., 2008). Additionally, peripheral human (Lengen et al., 2009) and central rodent (Neveu, 1990; Shen et al., 2005) data have shown differences in the immunological system, while additional monoamines (norepinephrine; Barnéoud et al., 1990) and enzymes (angiotensinases; Wu et al., 2010) were also associated with rodent pawedness. It has therefore been hypothesized that differences in this trait might be associated with other behavioral outcomes particularly cognition. Indeed, a small advantage of right-handed people in spatial ability has been reported (Somers et al., 2015) and pawedness/memory associations have been found in monkeys (Hopkins and Washburn, 1994) and mice (Wu et al., 2010). Furthermore, Prichard et al. (2013) reported that cognitive data is associated with the degree of handedness (and not its direction) as inconsistent handedness seems to be related with better episodic memory and improved belief updating/cognitive flexibility.

While handedness assessment in humans is simple, determination of associated behavioral and molecular differences poses several challenges: (i) the distribution of left- and righthanders in the population is uneven (Teng et al., 1976; Guadalupe et al., 2016) imposing the creation of specific left-enriched cohorts; (ii) social context may alter behavior (Teng et al., 1976), therefore increasing the percentage of strong right-handers and weak left-handers; and (iii) assessment of central molecular correlates is limited. The utilization of animal models became therefore very useful in this regard.

The Collins' (Collins, 1968) and the lateral paw preference tests (LPP; Waters and Denenberg, 1991) are amongst the most used tests to assess pawedness in rodents. The number of times a paw is used to retrieve food from an elevated tube and the amount of food retrieved from two lowered hoppers placed side-by-side are employed, respectively, as behavioral readouts. Despite the construct similarities, different results have been obtained between these two tests (Waters and Denenberg, 1991, 1994; Rogers and Bulman-Fleming, 1998) not only at the population level but, more importantly, at the individual level. Furthermore, both tests rely in the exclusive right/left paw use disregarding paw movement precision and possible intermediary strategies implicating the simultaneous use of both paws. Additionally, as measured by these methods, preference in rodents appears to a certain extant to be training/learning dependent (Collins, 1988; Stashkevich and Kulikov, 2001; Tang and Verstynen, 2002; Ribeiro et al., 2011).
To surpass these limitations, we have designed and validated an alternative test requiring minimal equipment for a fast determination of pawedness degree-the pawdeness trait test (PaTRaT). In this test, a circular grid separates the animal from a receptacle containing sugared items. Contrary to previous tests, the PaTRaT allows the simultaneous use of the two paws for reward handling reducing potential selection biases and making it more ethologically relevant. Also, the large size of the reward compared to the grid slits, imposes higher movement complexity for successful retrieval. Finally, the PaTRaT uses a classification system for dexterity degree that goes beyond the simple quantification of left-/right-paw retrievals. Additionally, we assessed potential associations between pawedness and behavioral outcomes, namely with impulsivity, memory and fine motor skills.

\section{MATERIALS AND METHODS}

\section{Animals}

Thirty male Sprague-Dawley rats (Charles-River Laboratories) with 6 months of age were kept under standard laboratory conditions: $12 \mathrm{~h}$ light/dark cycle (lights on at 8 a.m.), relative humidity of $55 \%, 22^{\circ} \mathrm{C}$ and ad libitum access to water. Food (4RF21, Mucedola SRL) was restricted to $1 \mathrm{~h}$ per day (last hour of the cycle light phase) during experimental protocols otherwise access was ad libitum. Body weight was controlled on a weekly basis to prevent weight losses superior to $15 \%$. Animals that failed to learn the PaTRaT in the training sessions (see below) were excluded from further analysis. Procedures involving animals were approved by local authorities and followed the EU Directive 2010/63/EU.

\section{Pawedness Trait Test (PaTRaT)}

\section{Apparatus}

The PaTRaT apparatus consisted of a custom-made plexiglass box open at the top. In the center, a metal wire mesh cylinder was used to confine the animal. Externally, accompanying cylinder's curvature, a plexiglass transparent piece was fixed to the bottom forming a receptacle for the rewards (Figure 1A).

\section{Experimental Protocol Training}

Two daily sessions were performed, separated by a minimum of $4 \mathrm{~h}$. Animals were habituated to the apparatus (1 session, $10 \mathrm{~min})$ and to the reward (Cheerios ${ }^{\circledR}$, Nestlé; 1 session, $10 \mathrm{~min}$ ). On the next sessions, animals were motivated to reach for the reward with their paws by placing single rewards close to the grid (1-2 sessions, $15 \mathrm{~min}$ ). The diameter of the reward was larger than the horizontal width of the metal mesh, increasing the demanding for successful retrievals.

\section{PaTRaT}

Four experimental sessions were performed, in which the animal had to retrieve 10 rewards in a maximum time of $10 \mathrm{~min}$ while the session was being recorded. Sessions were performed during 


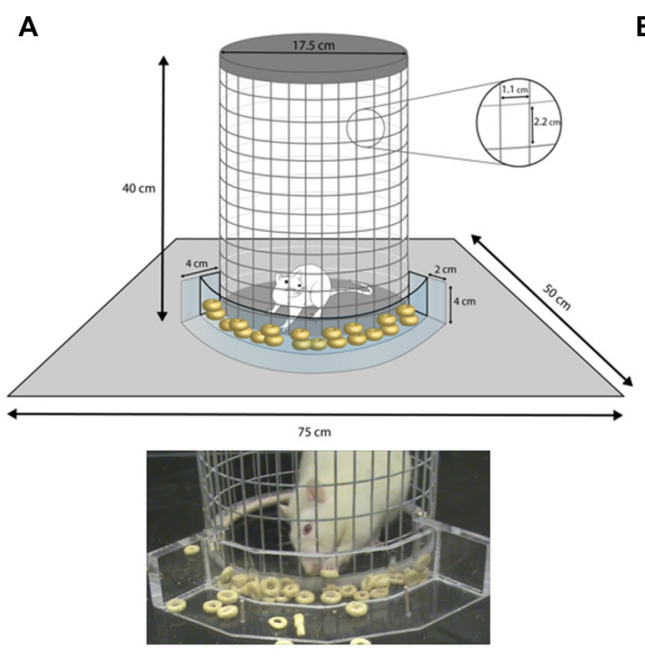

B

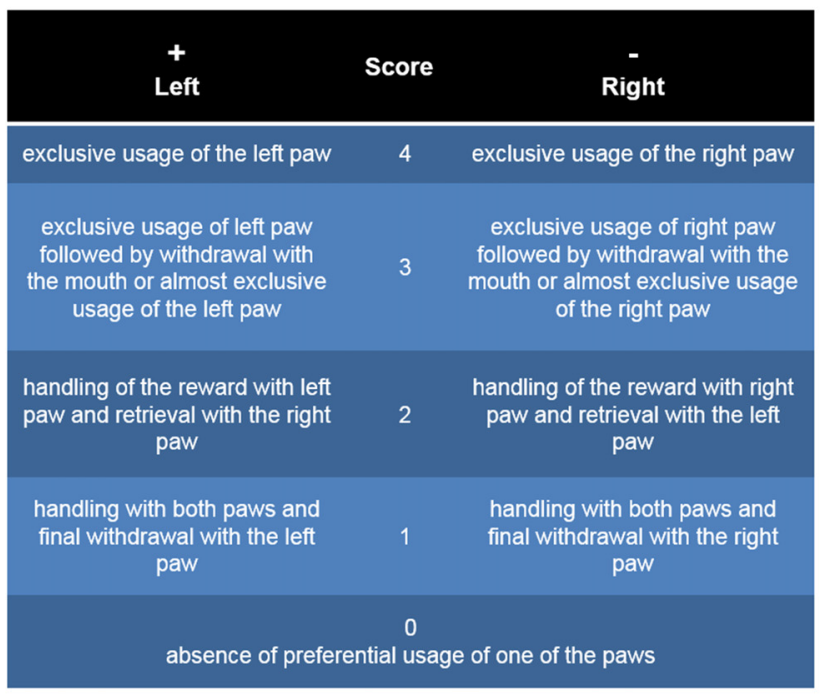

C

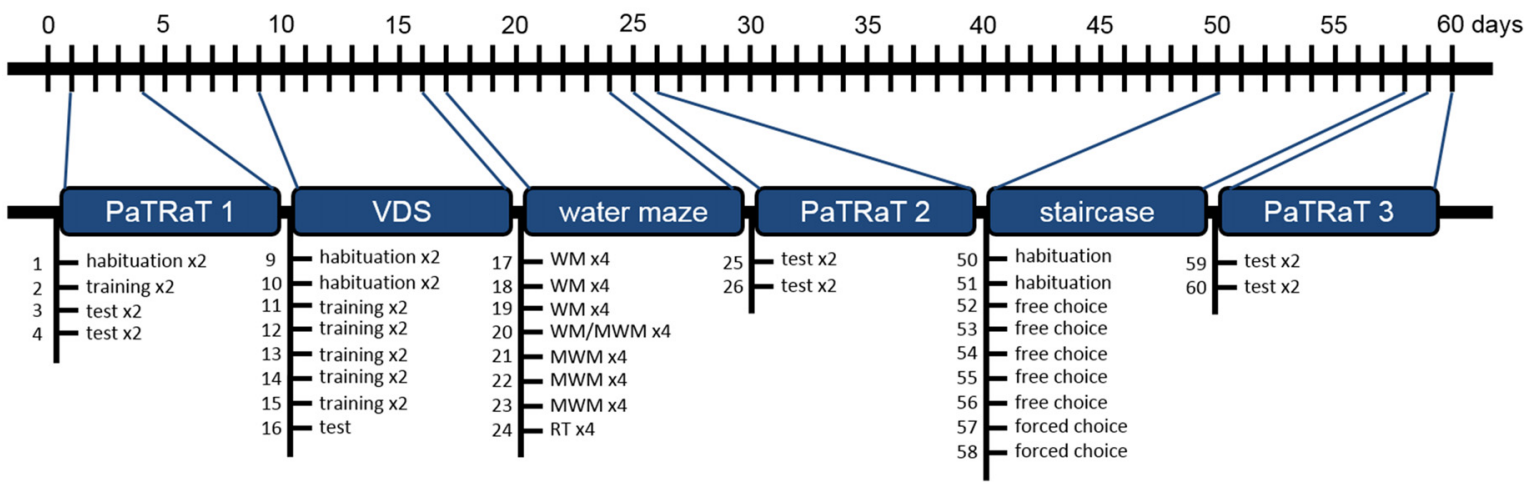

FIGURE 1 | Methods. (A) Scheme (top) and picture (bottom) depicting the pawedness apparatus. The animal is enclosed in a gridded cylinder. An exterior plexiglass structure contains the rewards. (B) Schematic table of classification for each retrieved reward. Positive and negative values correspond respectively to preferential usage of left and right paw and higher values are associated to more exclusive usage of said paw. (C) Timeline of behavioral analysis. Pawedness was assessed three times approximately 4 weeks apart. VDS and water mazes were performed between the two first evaluations and staircase test was performed before the third pawedness evaluation. Individual sessions are specified. VDS, Variable Delay-to-Signal; WM, working memory; MWM, Morris Water Maze; RT, reversal test.

the light period (8:00-12:00 and 14:00-18:00 for morning and afternoon sessions, respectively). Between animals the apparatus was cleaned with $10 \%$ ethanol.

To evaluate phenotype stability, two additional evaluations were performed 4 and 8 weeks after the first evaluation. Sessions were recorded and later evaluated independently by two observers. Additionally, the first evaluation was also rated by two inexperienced observers to assess inter-rater reliability.

\section{Behavioral rating}

Paw dexterity was determined by averaging the 40 trials (i.e., reward retrievals) of each evaluation. Each successful reward withdrawal was classified in a scale of +4 to -4 . Positive and negative values reflected preferential use of the left or right paw respectively and increasing values were associated with increasing preference. Classification was only attributed when a reward was retrieved; unsuccessful attempts were not rated. Rewards withdrawn without usage of any of the paws (i.e., with the mouth) were also not classified.
Score 4 corresponded to exclusive usage of the left paw; score 3 to exclusive usage of left paw followed by withdrawal with the mouth or to almost exclusive usage of the left paw (e.g., right paw used as support); score 2 was attributed when the animal handled the reward with its left paw but retrieved it with the right paw; and score 1 corresponded to handling with both paws and final withdrawal with the left paw. Score 0 was associated with absence of preferential usage of one of the paws, namely equal usage of both paws for reaching the reward and final withdrawal with the mouth. Symmetrical values corresponded to similar classification for the opposite paw (Figure 1B).

\section{Additional Behavioral Tests}

In order to assess potential lateral preference-behavior associations, other behaviors were assessed (Figure 1C).

\section{Variable Delay-to-Signal Test (VDS)}

The variable delay-to-signal (VDS) task was used to assess impulsive behavior. It was previously validated by drug-induced 
changes in impulsivity and by comparison with reference paradigms (Leite-Almeida et al., 2013). In short, the test was performed in a 5-hole operant chamber in which only the middle nosepoke aperture was available. Animals performed two daily sessions separated by a minimum of $5 \mathrm{~h}$ for a total of 8 days. There were four habituation sessions: the first two had the duration of $15 \mathrm{~min}$, lights were off, all nosepoke apertures were blocked and sugared pellets (45 mg, Bioserv Inc., Flemington, NJ, USA) were available at the food magazine. The latter two had the duration of $30 \mathrm{~min}$, all lights were on and pellets were available at both the food magazine and the nosepoke aperture.

Training consists of 10 sessions of 100 trials (or $30 \mathrm{~min}$ ) in which there was a $3 \mathrm{~s}$ ' interval between the beginning of the trial and the lightning of the nosepoke aperture (delay period). If a nosepoke was performed in this period, it was considered a premature response and it was punished with a $5 \mathrm{~s}$ ' timeout in complete darkness. If the nosepoke was performed in the $60 \mathrm{~s}$ ' period in which the light in the aperture was on, it was considered as a correct response and a reward was retrieved in the food magazine, initiating a new trial. If no nosepoke was performed, it was considered an omission and the animal was punished with a timeout.

Testing consisted of a single session at the end of the training. It was constituted by a total of 120 trials (or $90 \mathrm{~min}$ ), in which premature responses were not punished and the intervals were variable. It started with $253 \mathrm{~s}$ ' trials (3si), followed by $706 \mathrm{~s}$ (6 s) and 12 seconds' (12 s) (randomized) trials and again by $253 \mathrm{~s}$ ' trials (3sf). During the testing session multiple premature responses were allowed during the delay periods and the rate of premature responses per time of available delay was calculated (Leite-Almeida et al., 2013).

\section{Water Maze Test}

In order to test memory, animals were subjected to a modification of the Morris Water Maze (MWM) test (Morris, 1984), for which the procedures have been previously described (Cerqueira et al., 2007). Briefly, animals were placed in a black tank $170 \mathrm{~cm}$ in diameter filled with $31 \mathrm{~cm}$ of water at $22^{\circ} \mathrm{C}$. The tank was divided in four virtual quadrants, each associated with an external visual clue. A non-visible platform (black, $12 \mathrm{~cm}$ diameter, $30 \mathrm{~cm}$ high) was placed inside the tank and all movements were recorded through a video camera on the ceiling and tracked using a video-tracking system (Viewpoint, Champagne au mont d'or, France). In all trials the animal had $120 \mathrm{~s}$ to find the platform, at the end of which it was gently pushed towards if unable to complete the task. After reaching the platform, the animal was allowed $20 \mathrm{~s}$ on it before starting a new session.

Evaluation of working memory (WM) consisted of 4 days of evaluation, four sessions each. The position of the platform was maintained within each day, but changed on consecutive days, while the animal initiated each session on a different quadrant. The last day of WM evaluation was also the first of 4 days of MWM testing, in which the platform remained on the same place throughout all days of testing, while all remaining parameters were similar to the WM test. On the final day of testing, a reversal test (RT) was performed. Here, the platform (which had been in the same quadrant for 4 days) was moved to the opposite quadrant and four sessions equal to the ones above described were performed.

In all modalities, time to reach platform was evaluated. For WM and MWM, the average evolution curves throughout first to fourth trial or day were assessed respectively. For RT, the comparison was between time spent in the new and old quadrants.

\section{Staircase Test}

Aiming to assess potential differences in motor skills between left and right pawed animals, a modified version of the original staircase test (Montoya et al., 1991) was performed, for which most procedures have been previously described (Teixeira et al., 2017). This test required the usage of double staircase boxes (Campden Instruments, Lafayette, IN, USA), which consists of a narrow platform connected to a larger chamber with a moveable lid. A double removable seven-step staircase was inserted along both sides of the platform. In each session, five sugared pellets (45 mg, Bioserv Inc., Flemington, NJ, USA, EUA) were placed in each step and one daily session was performed. The first two sessions aim to habituate the animals and last respectively 5 and $10 \mathrm{~min}$ each, after which five test sessions were performed, each lasting $5 \mathrm{~min}$. The last two evaluations were forced choice sessions, in which only one of the staircases (left or right) had pellets in it. In all cases, at the end of the session, the remaining pellets were counted.

Measures of interest were reached level at each side (lowest level from which pellets were withdrawn) and success rate (number of pellets eaten/total number of pellets) in both normal and forced sessions.

\section{Statistical Analysis}

All statistical analyses were performed on Matlab R2009b software. $P<0.05$ was always considered the significance threshold. For assessment of inter-rater reliability Kappa statistics was used, namely Fleiss' Kappa when comparing between four observers and Cohen's Kappa when comparing the two experienced raters. Potential time-dependent differences in pawedness were assessed by comparing three separate time points using a repeated measures ANOVA.

For analysis of behavior mixed design ANOVAs were conducted. For VDS, the between subjects factor was pawedness group and the within subjects factor was session (training) or interval (test). For water maze, the between subjects factor was also pawedness group and the within subjects factor was either trial (WM), day (MWM) or quadrant (RT).

Differences in motor performance and motivation were assessed using simple group comparison. As normality could not be confirmed, non-parametric tests were used.

\section{RESULTS}

\section{Pawedness (Inter-Rater Agreement)}

Four observers (two experienced and two inexperienced) rated the first pawedness evaluation (Figure 2A). Inter-rater 
A

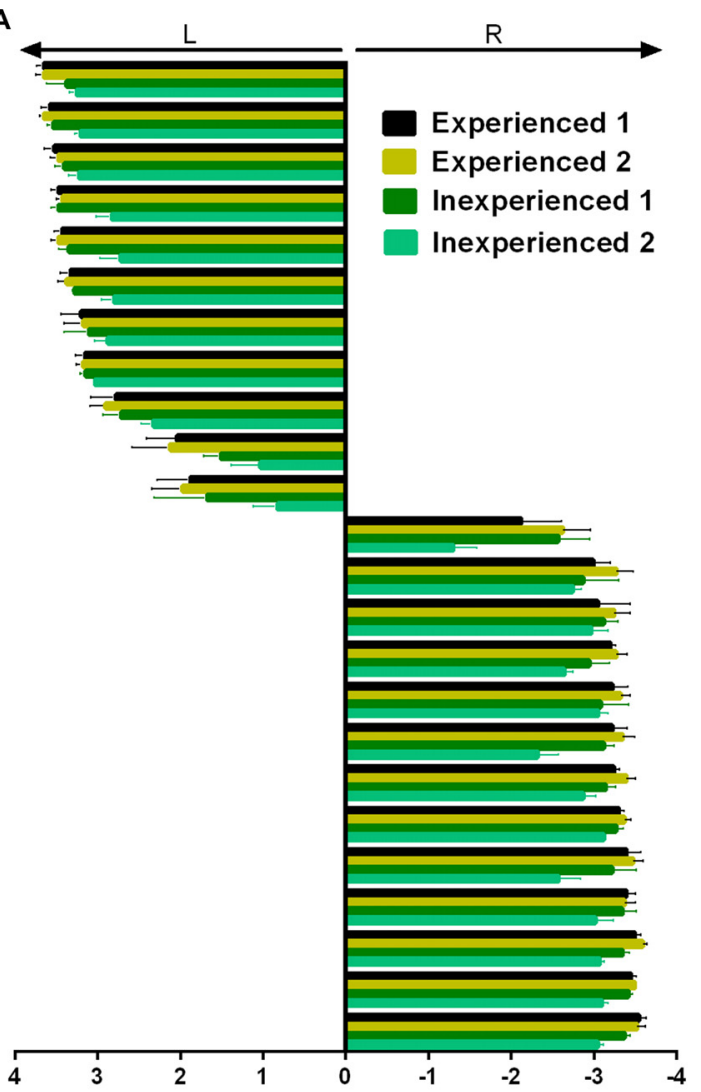

B

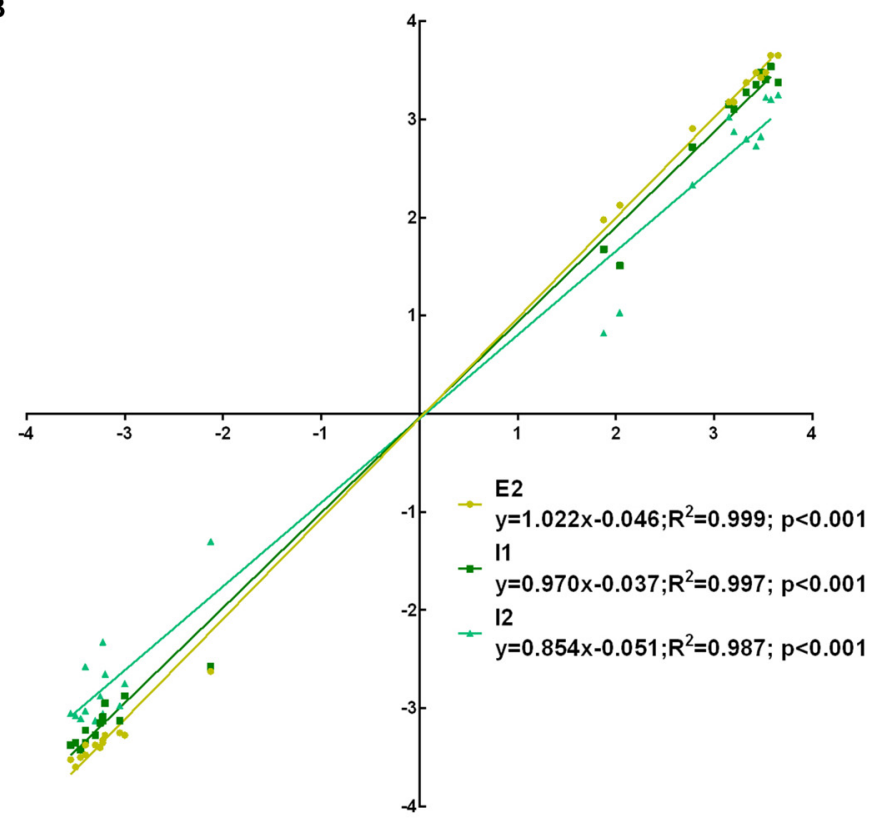

\begin{tabular}{ccccc|}
\hline & E1 & E2 & I1 & I2 \\
\hline E1 & 1 & 0.999 & 0.997 & 0.987 \\
E2 & 0.999 & 1 & 0.998 & 0.983 \\
I1 & 0.997 & 0.998 & 1 & 0.987 \\
I2 & 0.987 & 0.983 & 0.987 & 1 \\
\hline
\end{tabular}

FIGURE 2 | Pawdeness trait test (PaTRaT) rater comparison. Two experienced and two inexperienced raters scored the first pawedness evaluation.

(A) Representation of the score attributed by each rater for each animal shown as mean \pm SEM. (B) Experienced rater 1's scores correlated with scores attributed by all other raters (graph) and all raters show high correlation between them (table of $R^{2} \mathrm{~s}$ ). L, Left; R, Right; E, experienced; I, inexperienced.

agreement was assessed and rendered a "substantial agreement" (Fleiss' Kappa $=0.615 ; p<0.001 ; 95 \%$ CI $=0.590-0.640$ ). Additionally, individual raters' scores showed to be linearly correlated among them (Figure 2B).

\section{Pawedness (Temporal Stability)}

As the two experienced raters presented very high correlation and inter-rater agreement (Cohen's kappa $=0.932, p<0.001,95 \%$ $\mathrm{CI}=0.762-1.102$ ) on the first evaluation, this data was averaged between them and subsequent evaluations were assessed by both (2 sessions each) and averaged. Repeated measures ANOVA showed no effect of moment $(\mathrm{M})$ of evaluation (Figure 3A, $\left.F_{(2,44)}=0.641, p=0.532\right)$ and analysis of the logarithmic ratio between moments of evaluation showed no difference from 0 (Figure 3B, M2/M1: $Z=0.400, r=0.082, p=0.689$; M3/M2: $Z=1.156, r=0.241, p=0.248 ; \mathrm{M} 3 / \mathrm{M} 1: Z=1.247, r=0.260$, $p=0.212$ ).

Thus, from the total of 24 evaluated animals, all showed consistent paw preference across time. From these, 11 and 13, respectively showed left and right paw preference. Following veterinary decision one animal was excluded prior to M3. Up to this timepoint general aspects of well-being, weight and other behavioral measures were normal.

\section{Variable Delay-to-Signal (Impulsivity)}

During the learning phase, the evolution number of omissions across sessions (Figure 4A) showed an effect of session $\left(F_{(9,198)}=40.428, p<0.001\right)$, but no effect of pawedness group $\left(F_{(1,22)}=0.060, p=0.809\right)$ or interaction session/group $\left(F_{(9,198)}=0.690, p=0.718\right)$ indicating that both groups learned equally well the task. Similar results were found for correct nosepokes (session: $F_{(9,198)}=20.377, p<0.001$; group: $F_{(1,22)}=0.007, p=0.935$; interaction: $F_{(9,198)}=0.529$, $p=0.852$ ) and premature responses (Figure 4B-session: $F_{(9,198)}=38.350, p<0.001$; group: $F_{(1,22)}=0.005, p=0.943$; interaction: $\left.F_{(9,198)}=0.415, p=0.926\right)$. Left and right pawed animals thus showed no differences in learning the task.

Regarding impulsivity (Figure 4C), which is evaluated by delay intolerance (premature responses per $\min$ ), there was a significant effect of interval $\left(F_{(3,66)}=12.617, p<0.001\right)$ but no effect of group $\left(F_{(1,22)}=2.095, p=0.162\right)$ or interaction $\left(F_{(3,66)}=2.371, p=0.078\right)$. Similarly, $\log (3 \mathrm{sf} / 3 \mathrm{si})$ showed no 


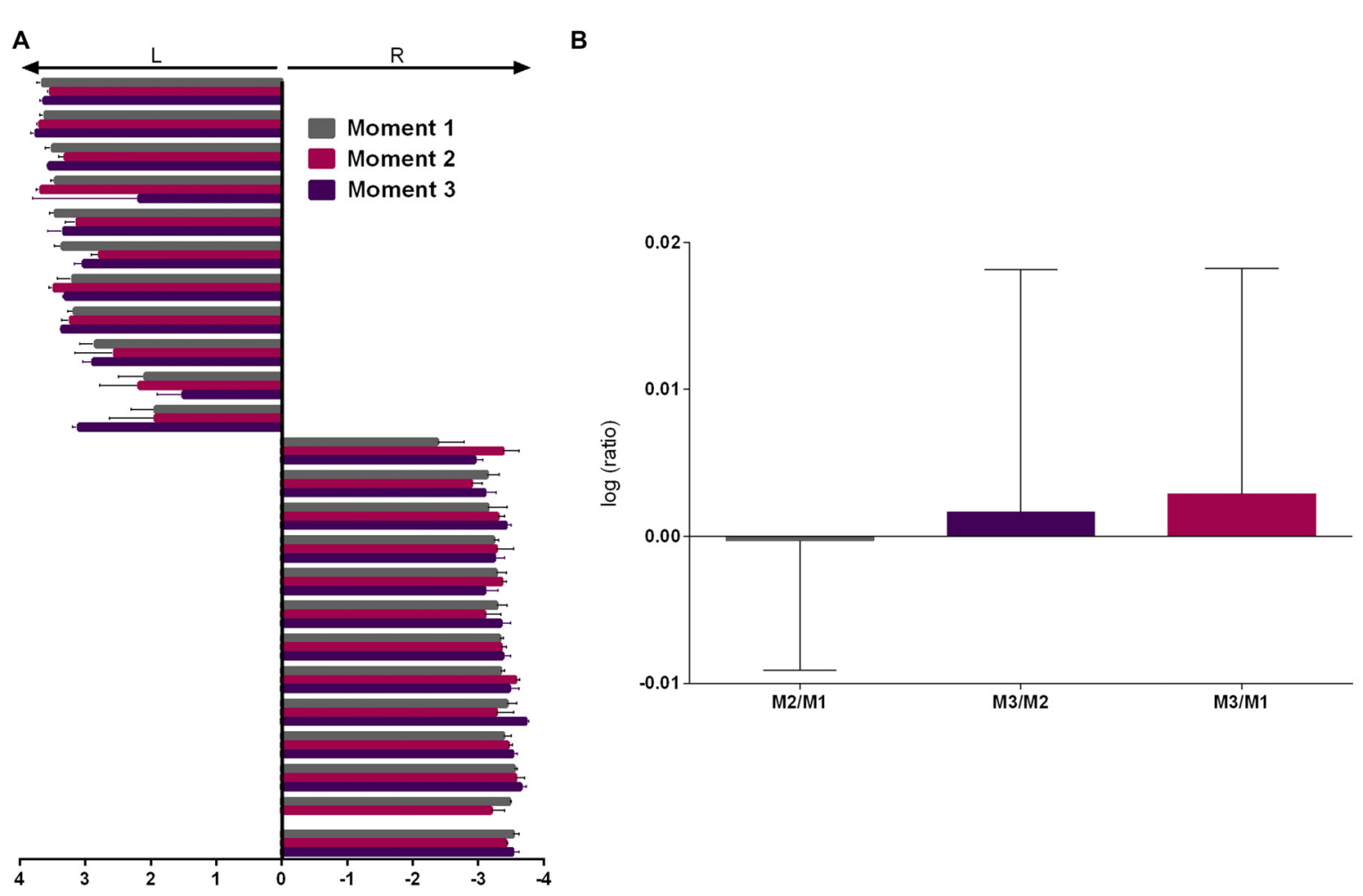

FIGURE 3 | PaTRaT time-related stability. Pawedness was evaluated at three separate moments. (A) Representation of the score attributed at each moment. $11 / 13$ animals showed left/right preference, respectively. (B) Graph shows the logarithmic ratios between the three moments of evaluation. Data is shown as mean \pm SEM. L, Left; R, Right; M, moment.

differences between animals with left and right paw preference (Figure 4D, $Z=0.758$ Cohen's $d=0.377, p=0.448$ ).

\section{Water Maze (Memory)}

The WM part of the water maze (Figure 5A) trial showed a significant effect on time to reach the platform $\left(F_{(3,66)}=8.487\right.$, $p<0.001)$, but no influences of pawedness group $\left(F_{(1,22)}=1.104\right.$, $p=0.305)$ or interaction $\left(F_{(3,66)}=0.373, p=0.773\right)$ were found. Similar data was found regarding MWM (Figure 5B-day: $F_{(3,66)}=1.075, p=0.001$; group: $F_{(1,22)}=0.001, p=0.974$; interaction: $\left.F_{(3,66)}=1.075, p=0.366\right)$. No effects were found on the RT component of the test (Figure 5C-quadrant: $F_{(1,22)}=1.291, p=0.268$; group: $F_{(1,22)}=0.387, p=0.540$; interaction: $\left.F_{(1,22)}=1.932, p=0.178\right)$. In all cases, similar results were found when analyzing distance traveled rather than time to reach the platform (data not shown).

\section{Fine Motor and Locomotor Performance}

The staircase test showed no group differences regarding fine motor coordination for both right (level normal: $Z=0.434$ Cohen's $d=0.105, p=0.664$; level forced: $Z=1.074$ Cohen's $d=0.110, p=0.283$; success rate normal: $Z=0.838$ Cohen's $d=0.334, p=0.402$; success rate forced: $Z=1.131$ Cohen's $d=0.455, p=0.258$ ) or left (level normal: $Z=0.203$ Cohen's $d=0.073, p=0.839$; level forced:
$Z=0.602$ Cohen's $d=0.117, p=0.548$; success rate normal: $Z=0.260$ Cohen's $d=0.177, p=0.795$; success rate forced: $Z=1.389$ Cohen's $d=0.176, p=0.165)$ paws (Figure 6A).

Regarding latency to reward retrieval in the VDS no differences between animals with left or right paw preference were found ( $Z=0.513$ Cohen's $d=0.124, p=0.608$ ), as seen through the time to retrieve the reward during the VDS test (Figure 6B). Additionally, no differences were found in the water maze average swimming velocity $(Z=0.051$ Cohen's $d=0.486$, $p=0.959 ;$ Figure 6C).

\section{DISCUSSION}

PaTRaT is a grading system to evaluate pawedness in rats. It is of simple implementation, requiring minimal equipment. Also, it has high inter-rater reliability and temporal stability of the outcomes. The PaTRaT displays several advantages over the most used tests-the Collins' (Collins, 1968) and LPP (Waters and Denenberg, 1991) tests, namely: (i) the reward can be readily seen, smelled and touched by the animals, increasing motivation. Indeed, we observed in preliminary assays that animals were more prone to perform the test when a greater amount of reward $\left(\approx 50\right.$ vs. $\approx 20$ cheerios $\left.{ }^{\circledR}\right)$ was available (data not shown); (ii) the apparatus imposes no constraints to the simultaneous use of both paws for reward manipulation, 

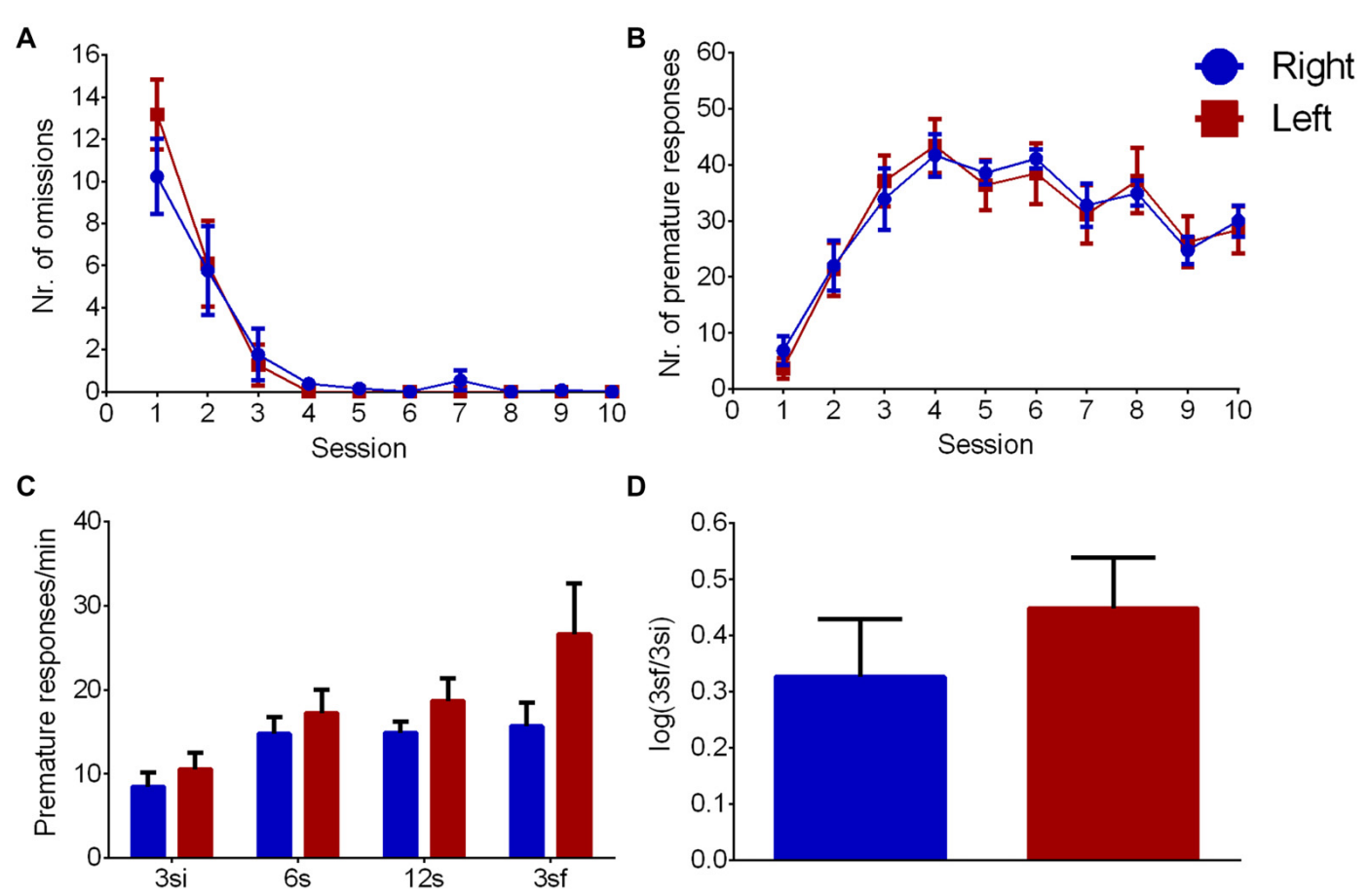

D

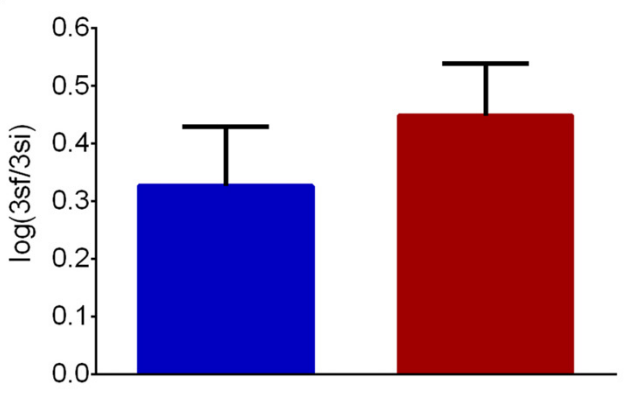

FIGURE 4 | Left/Right differences in impulsivity. Group comparison during VDS training (A,B) and test (C,D). (A) Number of omissions, (B) number of premature responses, (C) premature responses per minute per interval and (D) logarithmic ratio of the number of premature responses in 3sf and 3si intervals. Data is shown as mean \pm SEM. Blue-animals with right paw preference; Red-animals with left paw preference.

A

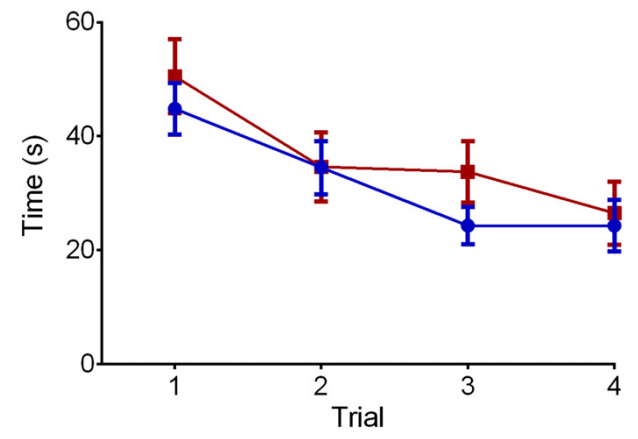

C

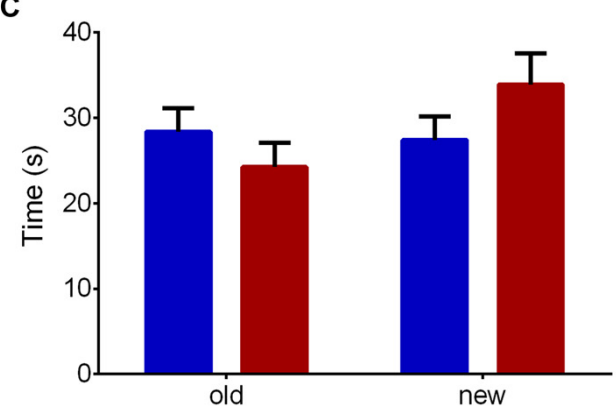

B

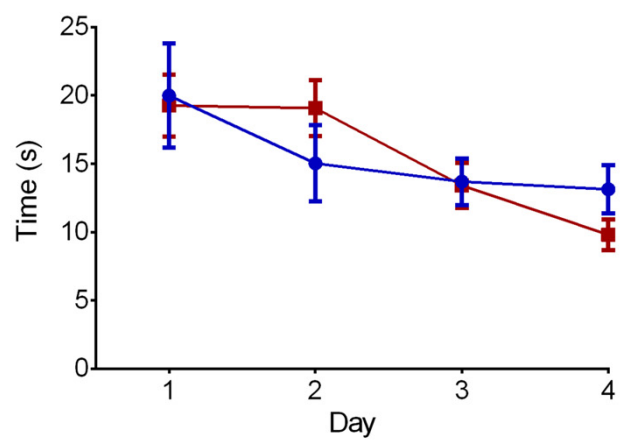

\section{Right}

Left

FIGURE 5 | Group effects on memory. Group comparison on the water maze test in WM (A), classic Morris (B) and reversal (C) components. Data is shown as mean \pm SEM. Blue-animals with right paw preference; Red-animals with left paw preference. 


\section{A}

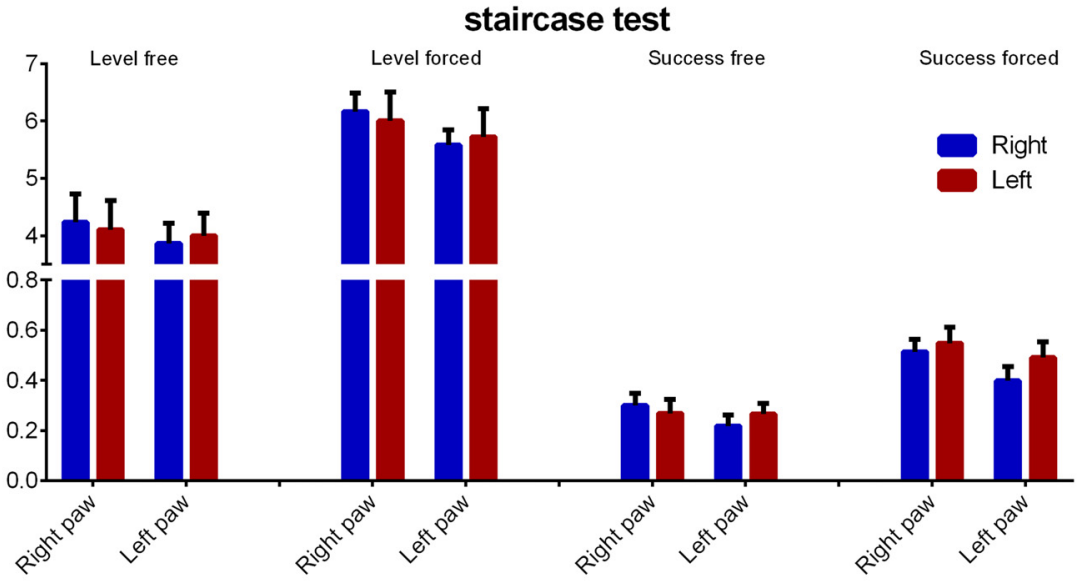

B

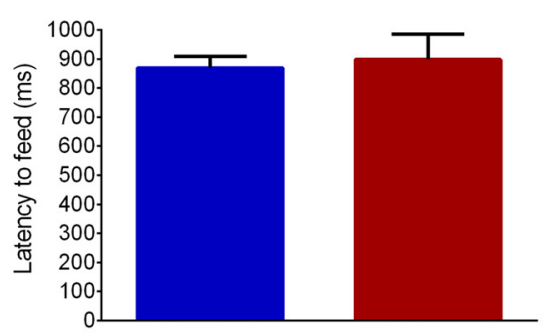

C

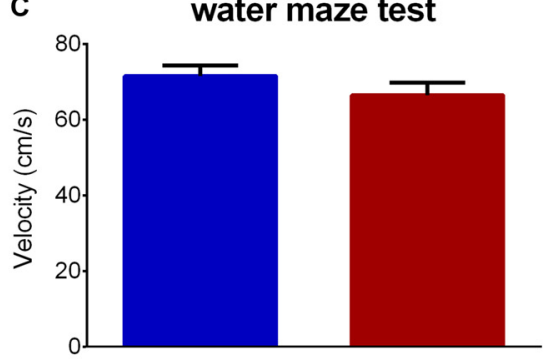

FIGURE 6 | Fine motor and locomotor performance. Group comparison regarding fine motor coordination (A-staircase test), motivation to eat (B-latency to feed in the VDS test) and motor performance (C-velocity in the water maze). Data is shown as mean \pm SEM. VDS, Variable Delay-to-Signal; Blue-animals with right paw preference; Red-animals with left paw preference.

decreasing potential selection biases (Ribeiro et al., 2011) and providing a more ethological setting; (iii) the large reward size relative to the mesh grid imposes higher dexterity, i.e., a sequence of relatively complex movements is required for a successful retrieval of the reward; (iv) assessment is based on four independent sessions (10 trials each) avoiding potential confounders as limb alternation due to tiredness and satiation (Bulman-Fleming et al., 1997); (v) the grading system is of simple application and has a high inter-rater agreement even among inexperienced observers; and finally (vi) PaTRaT side preference index, contrary to the simple quantification of left/right paw retrievals, accounts for intermediate pawedness and is therefore more akin to human handedness assessment (Prichard et al., 2013). Additionally, two sessions were enough to achieve a sustained performance in $80 \%$ of the animals. In our study, the prevalence of right- and left-pawed rats was similar (54\% vs. $46 \%$, respectively), which is in accordance with a previous report (Stashkevich and Kulikov, 2001) but in opposition with others that report a rightward bias up to $\approx 82.4 \%$ (Pençe, 2002; Elalmis et al., 2003; Güven et al., 2003; Wu et al., 2010). Scores were stable across the three evaluations even when other manipulations/behavioral paradigms were interposed; potential differences in fine motricity (or even in general motor performance) were experimentally excluded. Importantly, we evaluated the PaTRaT in male Sprague-Dawley rats but strain, sex and species differences have been described in the context of paw preference protocols (see for example Betancur et al., 1991).

It has been argued that a less marked lateralization in rodents (or other animals) in comparison with humans results from the fact that pawedness assessments rely on the evaluation of simple movements (e.g., grabbing food) instead of fine movements as in humans (see for review on nonhuman primates, Hopkins, 2013). Thus, the increased movement complexity of the PaTRaT assay may explain the absence of ambidextrous animals, normally reported as being 7.4\%-23\% (Stashkevich and Kulikov, 2001; Pençe, 2002; Elalmis et al., 2003; Güven et al., 2003; Wu et al., 2010), as it allows a better separation of left- and rightpawed animals (i.e., average scores close to the limits of the scale). In fact, decreases in the PaTRaT absolute scores were mostly related with poorer performances of the preferred paw and not with retrievals with the non-preferred paw (practically absent).

Pawedness in rats has been associated with central asymmetries in monoamines, notably dopamine and other molecular players (Barnéoud et al., 1990; Cabib et al., 1995; Budilin et al., 2008). These have been hypothesized to underlie associations between pawedness direction (and/or magnitude) and performance in several behavioral domains (Wu et al., 2010)_see also Prichard et al. (2013) for a comprehensive review in human studies. Specifically regarding impulsive behavior, several studies have demonstrated an influence of 
the dopamine levels (see for review, Dalley and Roiser, 2012; D'Amour-Horvat and Leyton, 2014). We have nevertheless observed no differences between left- and right-biased animals on impulsive behavior both on the learning protocol or in the VDS test. Additionally, no differences were observed in long-term and working spatial reference memories and reversal learning.

In conclusion, the PaTRaT is a simple, inexpensive and reliable test for assessment of pawedness degree and direction in rats. It relies on a grading system for hind paw use and has high inter-rater agreement (even for inexperienced observers). At the population level, we observed a nearly equal distribution of left- and right-biased rats and individual preferences were stable across sessions.

\section{AUTHOR CONTRIBUTIONS}

AMC, ME and HL-A designed the experiment; AMC, ME and MRG performed the experiments, AMC, ME, SPN and SB rated the animals; $\mathrm{ME}$ and HL-A performed the statistical analysis; AMC, ME, NS, AA and HL-A analyzed and interpreted the

\section{REFERENCES}

Ambeskovic, M., Soltanpour, N., Falkenberg, E. A., Zucchi, F. C. R., Kolb, B., and Metz, G. A. S. (2017). Ancestral exposure to stress generates new behavioral traits and a functional hemispheric dominance shift. Cereb. Cortex 27, 2126-2138. doi: 10.1093/cercor/bhw063

Barnéoud, P., le Moal, M., and Neveu, P. J. (1990). Asymmetric distribution of brain monoamines in left- and right-handed mice. Brain Res. 520, 317-321. doi: 10.1016/0006-8993(90)91721-r

Betancur, C., Neveu, P. J., and Le Moal, M. (1991). Strain and sex differences in the degree of paw preference in mice. Behav. Brain Res. 45, 97-101. doi: 10.1016/s0166-4328(05)80185-8

Budilin, S. Y., Midzyanovskaya, I. S., Shchegolevskii, N. V., Ioffe, M. E., and Bazyan, A. S. (2008). Asymmetry in dopamine levels in the nucleus accumbens and motor preference in rats. Neurosci. Behav. Physiol. 38, 991-994. doi: 10.1007/s11055-008-9082-6

Bulman-Fleming, M. B., Bryden, M. P., and Rogers, T. T. (1997). Mouse paw preference: effects of variations in testing protocol. Behav. Brain Res. 86, 79-87. doi: 10.1016/s0166-4328(96)02249-8

Cabib, S., D’Amato, F. R., Neveu, P. J., Deleplanque, B., Le Moal, M., and Puglisi-Allegra, S. (1995). Paw preference and brain dopamine asymmetries. Neuroscience 64, 427-432. doi: 10.1016/0306-4522(94)00401-p

Cerqueira, J. J., Mailliet, F., Almeida, O. F., Jay, T. M., and Sousa, N. (2007). The prefrontal cortex as a key target of the maladaptive response to stress. J. Neurosci. 27, 2781-2787. doi: 10.1523/JNEUROSCI.4372-06.2007

Collins, R. L. (1968). On the inheritance of handedness. I. Laterality in inbred mice. J. Hered. 59, 9-12. doi: 10.1093/oxfordjournals.jhered.a107656

Collins, R. L. (1988). Observational learning of a left-right behavioral asymmetry in mice (Mus musculus). J. Comp. Psychol. 102, 222-224. doi: 10.1037/07357036.102.3.222

Dalley, J. W., and Roiser, J. P. (2012). Dopamine, serotonin and impulsivity. Neuroscience 215, 42-58. doi: 10.1016/j.neuroscience.2012.03.065

D'Amour-Horvat, V., and Leyton, M. (2014). Impulsive actions and choices in laboratory animals and humans: effects of high vs. low dopamine states produced by systemic treatments given to neurologically intact subjects. Front. Behav. Neurosci. 8:432. doi: 10.3389/fnbeh.2014. 00432

de la Fuente-Fernández, R., Kishore, A., Calne, D. B., Ruth, T. J., and Stoessl, A. J. (2000). Nigrostriatal dopamine system and motor lateralization. Behav. Brain Res. 112, 63-68. doi: 10.1016/s0166-4328(00)00165-0 data; AMC, ME and HL-A wrote the article's initial version. All authors revised and approved the final version of the manuscript.

\section{FUNDING}

This work has been funded by the European Regional Development Fund (FEDER), through the Competitiveness Factors Operational Programme (COMPETE) and the Northern Portugal Regional Operational Programme (NORTE 2020) under the Portugal 2020 Partnership Agreement (project NORTE-01-0145-FEDER-000023). It was also funded by National funds, through the Foundation for Science and Technology (Fundação para a Ciência e a Tecnologia, FCT), under the scope of the projects POCI-01-0145-FEDER007038 and PTDC/NEU-SCC/5301/2014. Researchers were supported by FCT grant numbers SFRH/BD/109111/2015 (AMC), SFRH/BD/52291/2013 (ME via Inter-University Doctoral Programme in Ageing and Chronic Disease, PhDOC), $\mathrm{PD} / \mathrm{BD} / 114120 / 2015$ (SPN via PhDOC), SFRH/BD/89936/2012 (SB), $\mathrm{PD} / \mathrm{BD} / 114117 / 2015$ (MRG via PhDOC) and SFRH/BPD/80118/2011 (HL-A).

Elalmis, D. D., Ozgünen, K. T., Binokay, S., Tan, M., Ozgünen, T., and Tan, U. (2003). Differential contributions of right and left brains to paw skill in right- and left-pawed female rats. Int. J. Neurosci. 113, 1023-1042. doi: 10.1080/00207450390204068

Galaburda, A. M., LeMay, M., Kemper, T. L., and Geschwind, N. (1978). Rightleft asymmetrics in the brain. Science 199, 852-856. doi: 10.1126/science. 341314

Good, C. D., Johnsrude, I., Ashburner, J., Henson, R. N., Friston, K. J., and Frackowiak, R. S. (2001). Cerebral asymmetry and the effects of sex and handedness on brain structure: a voxel-based morphometric analysis of 465 normal adult human brains. Neuroimage 14, 685-700. doi: 10.1006/nimg. 2001.0857

Guadalupe, T., Mathias, S. R., vanErp, T. G., Whelan, C. D., Zwiers, M. P., Abe, Y., et al. (2016). Human subcortical brain asymmetries in 15,847 people worldwide reveal effects of age and sex. Brain Imaging Behav. 1-18. doi: 10.1007/s11682016-9629-z [Epub ahead of print].

Guadalupe, T., Willems, R. M., Zwiers, M. P., Arias Vasquez, A., Hoogman, M., Hagoort, P., et al. (2014). Differences in cerebral cortical anatomy of left- and right-handers. Front. Psychol. 5:261. doi: 10.3389/fpsyg.2014.00261

Güven, M., Elalmiş, D. D., Binokay, S., and Tan, U. (2003). Population-level right-paw preference in rats assessed by a new computerized food-reaching test. Int. J. Neurosci. 113, 1675-1689. doi: 10.1080/00207450390249258

Hopkins, W. D. (2013). Comparing human and nonhuman primate handedness: challenges and a modest proposal for consensus. Dev. Psychobiol. 55, 621-636. doi: 10.1002/dev.21139

Hopkins, W. D., and Washburn, D. A. (1994). Do right- and left-handed monkeys differ on cognitive measures? Behav. Neurosci. 108, 1207-1212. doi: 10.1037/0735-7044.108.6.1207

Hugdahl, K. (2011). Hemispheric asymmetry: contributions from brain imaging. Wiley Interdiscip. Rev. Cogn. Sci. 2, 461-478. doi: 10.1002/wcs.122

Leite-Almeida, H., Melo, A., Pêgo, J. M., Bernardo, S., Milhazes, N., Borges, F., et al. (2013). Variable delay-to-signal: a fast paradigm for assessment of aspects of impulsivity in rats. Front. Behav. Neurosci. 7:154. doi: 10.3389/fnbeh.2013. 00154

Lengen, C., Regard, M., Joller, H., Landis, T., and Lalive, P. (2009). Anomalous brain dominance and the immune system: do left-handers have specific immunological patterns? Brain Cogn. 69, 188-193. doi: 10.1016/j.bandc.2008. 07.008

Montoya, C. P., Campbell-Hope, L. J., Pemberton, K. D., and Dunnett, S. B. (1991). The "staircase test": a measure of independent forelimb reaching and 
grasping abilities in rats. J. Neurosci. Methods 36, 219-228. doi: 10.1016/01650270(91)90048-5

Morris, R. (1984). Developments of a water-maze procedure for studying spatial learning in the rat. J. Neurosci. Methods 11, 47-60. doi: 10.1016/01650270(84)90007-4

Narr, K. L., Bilder, R. M., Luders, E., Thompson, P. M., Woods, R. P., Robinson, D., et al. (2007). Asymmetries of cortical shape: effects of handedness, sex and schizophrenia. Neuroimage 34, 939-948. doi: 10.1016/j.neuroimage.2006. 08.052

Neveu, P. J. (1990). Lymphocyte responsiveness and autoantibody production are associated with a functional brain asymmetry in mice. Int. J. Neurosci. 51, 331-333. doi: 10.3109/00207459008999735

Nielsen, D. M., Visker, K. E., Cunningham, M. J., Keller, R. W. Jr., Glick, S. D., and Carlson, J. N. (1997). Paw preference, rotation, and dopamine function in Collins HI and LO mouse strains. Physiol. Behav. 61, 525-535. doi: 10.1016/s0031-9384(96)00496-9

Ocklenburg, S., Friedrich, P., Güntürkün, O., and Genç, E. (2016). Voxel-wise grey matter asymmetry analysis in left- and right-handers. Neurosci. Lett. 633, 210-214. doi: 10.1016/j.neulet.2016.09.046

Pençe, S. (2002). Paw preference in rats. J. Basic Clin. Physiol. Pharmacol. 13, 41-49. doi: 10.1515/jbcpp.2002.13.1.41

Prichard, E., Propper, R. E., and Christman, S. D. (2013). Degree of handedness, but not direction, is a systematic predictor of cognitive performance. Front. Psychol. 4:9. doi: 10.3389/fpsyg.2013.00009

Ribeiro, A. S., Eales, B. A., and Biddle, F. G. (2011). Learning of paw preference in mice is strain dependent, gradual and based on short-term memory of previous reaches. Anim. Behav. 81, 249-257. doi: 10.1016/j.anbehav.2010. 10.014

Rogers, L. J. (2009). Hand paw preferences in relation to the lateralized brain. Philos. Trans. R. Soc. Lond. B Biol. Sci. 364, 943-954. doi: 10.1098/rstb. 2008.0225

Rogers, L. J. (2014). Asymmetry of brain and behavior in animals: its development, function, and human relevance. Genesis 52, 555-571. doi: 10.1002/dvg.22741

Rogers, T. T., and Bulman-Fleming, M. B. (1998). Arousal mediates relations among medial paw preference, lateral paw preference, and spatial preference in the mouse. Behav. Brain Res. 93, 51-62. doi: 10.1016/s0166-4328(97) 00141-1

Schwarting, R., Nagel, J. A., and Huston, J. P. (1987). Asymmetries of brain dopamine metabolism related to conditioned paw usage in the rat. Brain Res. 417, 75-84. doi: 10.1016/0006-8993(87)90181-8

Shen, Y. Q., Hébert, G., Moze, E., Li, K. S., and Neveu, P. J. (2005). Asymmetrical distribution of brain interleukin-6 depends on lateralization in mice. Neuroimmunomodulation 12, 189-194. doi: 10.1159/000 084852
Somers, M., Shields, L. S., Boks, M. P., Kahn, R. S., and Sommer, I. E. (2015). Cognitive benefits of right-handedness: a meta-analysis. Neurosci. Biobehav. Rev. 51, 48-63. doi: 10.1016/j.neubiorev.2015.01.003

Stashkevich, I. S., and Kulikov, M. A. (2001). The formation of lateralized motor habits in rats. Neurosci. Behav. Physiol. 31, 269-274. doi: 10.1023/A:1010378316821

Sun, T., and Walsh, C. A. (2006). Molecular approaches to brain asymmetry and handedness. Nat. Rev. Neurosci. 7, 655-662. doi: 10.1038/nrn1930

Tang, A. C., and Verstynen, T. (2002). Early life environment modulates 'handedness' in rats. Behav. Brain Res. 131, 1-7. doi: 10.1016/s01664328(01)00330-8

Teixeira, F. G., Carvalho, M. M., Panchalingam, K. M., Rodrigues, A. J., MendesPinheiro, B., Anjo, S., et al. (2017). Impact of the secretome of human mesenchymal stem cells on brain structure and animal behavior in a rat model of Parkinson's disease. Stem Cells Transl. Med. 6, 634-646. doi: 10.5966/sctm. 2016-0071

Teng, E. L., Lee, P. H., Yang, K. S., and Chang, P. C. (1976). Handedness in a Chinese population: biological, social, and pathological factors. Science 193, 1148-1150. doi: 10.1126/science.986686

Toga, A. W., and Thompson, P. M. (2003). Mapping brain asymmetry. Nat. Rev. Neurosci. 4, 37-48. doi: 10.1038/nrn1009

Uguru-Okorie, D. C., and Arbuthnott, G. W. (1981). Altered paw preference after unilateral 6-hydroxy-dopamine injections into lateral hypothalamus. Neuropsychologia 19, 463-467. doi: 10.1016/0028-3932(81)90077-4

Waters, N. S., and Denenberg, V. H. (1991). A measure of lateral paw preference in the mouse. Physiol. Behav. 50, 853-856. doi: 10.1016/0031-9384(91)90030-r

Waters, N. S., and Denenberg, V. H. (1994). Analysis of two measures of paw preference in a large population of inbred mice. Behav. Brain Res. 63, 195-204. doi: 10.1016/0166-4328(94)90091-4

Wu, H. M., Wang, C., Wang, X. L., Wang, L., Chang, C. W., Wang, P., et al. (2010). Correlations between angiotensinase activity asymmetries in the brain and paw preference in rats. Neuropeptides 44, 253-259. doi: 10.1016/j.npep.2009.12.016

Conflict of Interest Statement: The authors declare that the research was conducted in the absence of any commercial or financial relationships that could be construed as a potential conflict of interest.

Copyright (C) 2017 Cunha, Esteves, das Neves, Borges, Guimarães, Sousa, Almeida and Leite-Almeida. This is an open-access article distributed under the terms of the Creative Commons Attribution License (CC BY). The use, distribution or reproduction in other forums is permitted, provided the original author(s) or licensor are credited and that the original publication in this journal is cited, in accordance with accepted academic practice. No use, distribution or reproduction is permitted which does not comply with these terms. 